\title{
Editorial
}

\section{Intelligent Fault Diagnosis Based on Vibration Signal Analysis}

\author{
Minvydas Ragulskis, ${ }^{1}$ Lu Chen, ${ }^{2}$ Ganging Song, ${ }^{3}$ and Ameen El Sinawi ${ }^{4}$ \\ ${ }^{1}$ Department of Mathematical Modelling, Kaunas University of Technology, LT-51368 Kaunas, Lithuania \\ ${ }^{2}$ School of Reliability and Systems Engineering, Beihang University, Beijing 100191, China \\ ${ }^{3}$ Department of Mechanical Engineering, University of Houston, Houston, TX 77204, USA \\ ${ }^{4}$ Department of Mechanical Engineering, The Petroleum Institute, Abu Dhabi, UAE
}

Correspondence should be addressed to Minvydas Ragulskis; minvydas.ragulskis@ktu.lt

Received 30 January 2017; Accepted 30 January 2017; Published 19 February 2017

Copyright (C) 2017 Minvydas Ragulskis et al. This is an open access article distributed under the Creative Commons Attribution License, which permits unrestricted use, distribution, and reproduction in any medium, provided the original work is properly cited.

Intelligent fault diagnosis has attracted increasing attention during the past decades in various industrial fields, such as aerospace, shipbuilding, manufacturing, sustainable energy, infrastructure development, and transportation. Preventive fault diagnosis is expected to improve machinery operational reliability and safety for complicated systems or equipment, further reducing the cost of cycle life and avoiding system risk. Nowadays, many diagnostic applications are deployed based on the vibration signal analysis. Vibration signals can be conveniently acquired and contain abundant signature information that reflects the potential failures and performance degradation trends of the monitored system. This special issue aims to publish new progress with the state of the art in the various engineering applications and provides a platform for researchers and engineers to share their novel theories and methodologies so as to improve technologies in the research area of intelligent fault diagnosis.

This special issue on intelligent fault diagnosis based on vibration signal analysis offers a unique opportunity to present the latest trends and state-of-the-art research in this rapidly developing field of research. 9 of 23 papers are dedicated for developing new techniques for bearing fault diagnosis. Enhanced $k$-NN classifiers, sensitive selection of intrinsic model functions, adaptive stochastic resonance analysis algorithms, lifting wavelet packet symbolic entropy assessment, cut-edge weight confidence intervals based on tri-training, advanced image recognition algorithms in the EEMD space, STFT-based deep learning techniques, and deep belief networks coupled with multisensor information fusion algorithms are just a few advanced approaches represented in this special issue in the area of rolling bearing diagnosis.

Other papers of the special issue are dedicated to other (but not less important) areas or application: intelligent fault diagnosis in gearboxes, wind turbines, hydraulic pumps, turbo generators, electronic devices, vibrations isolators, machine tools, and so forth. Recursive estimation of kernel ridge regression, advanced $K$-means and multiclass SVM classifiers, modified time-frequency Shannon entropy method, and advanced image analysis techniques in frequency spaces are just a few novel techniques and algorithms used for intelligent fault diagnosis in these demanding applications.

We hope that this special issue will be beneficial for specialists, graduate students, researchers, and scientists working in the rapidly developing area of intelligent fault diagnosis based on vibration signal analysis.

\section{Acknowledgments}

The editors of the special issue would like to thank authors of all submitted papers.

$$
\begin{array}{r}
\text { Minvydas Ragulskis } \\
\text { Lu Chen } \\
\text { Ganging Song } \\
\text { Ameen El Sinawi }
\end{array}
$$




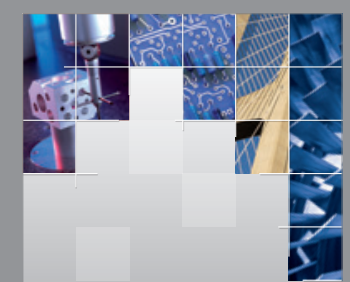

\section{Enfincering}
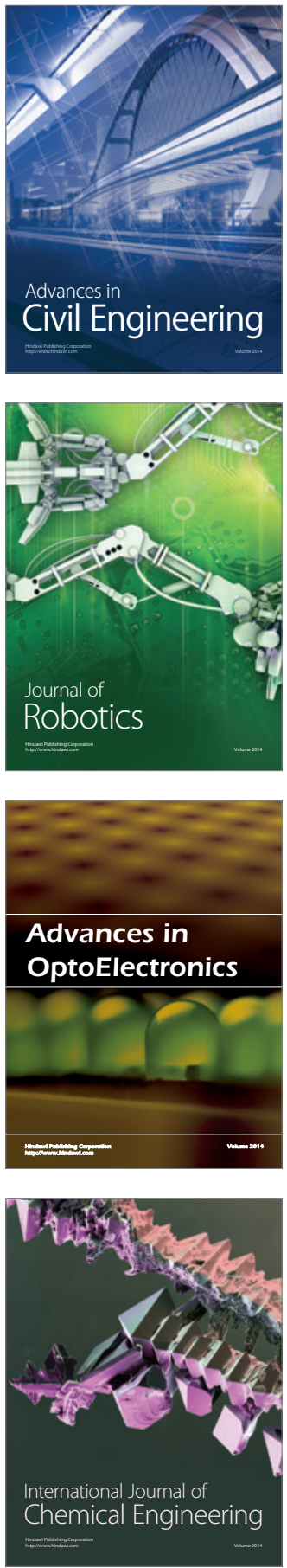

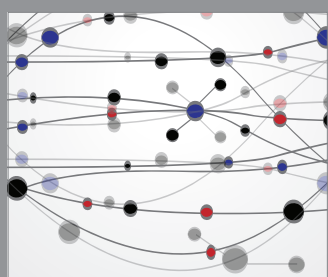

The Scientific World Journal

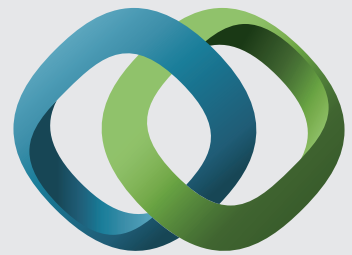

\section{Hindawi}

Submit your manuscripts at

https://www.hindawi.com
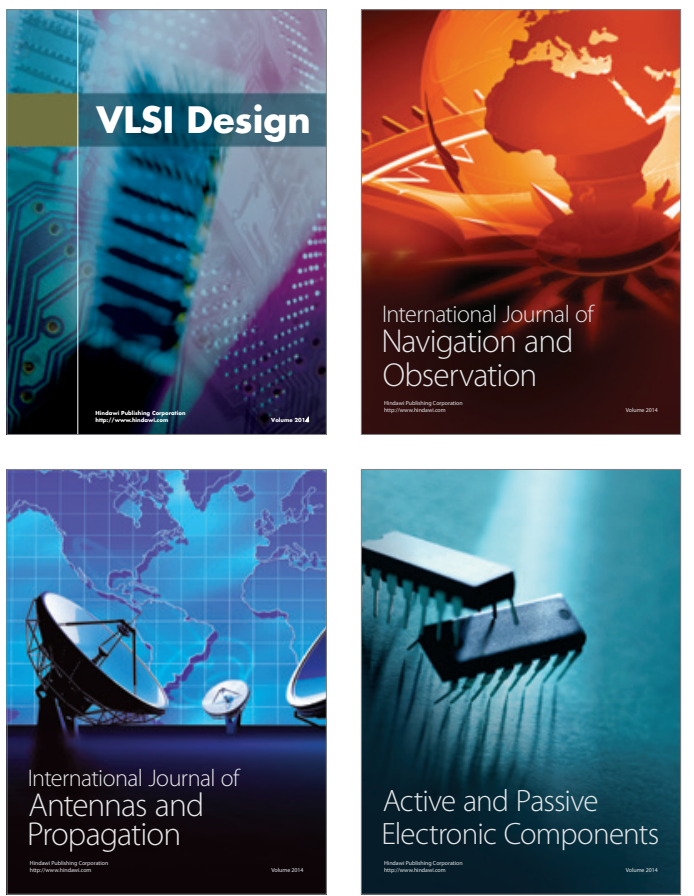
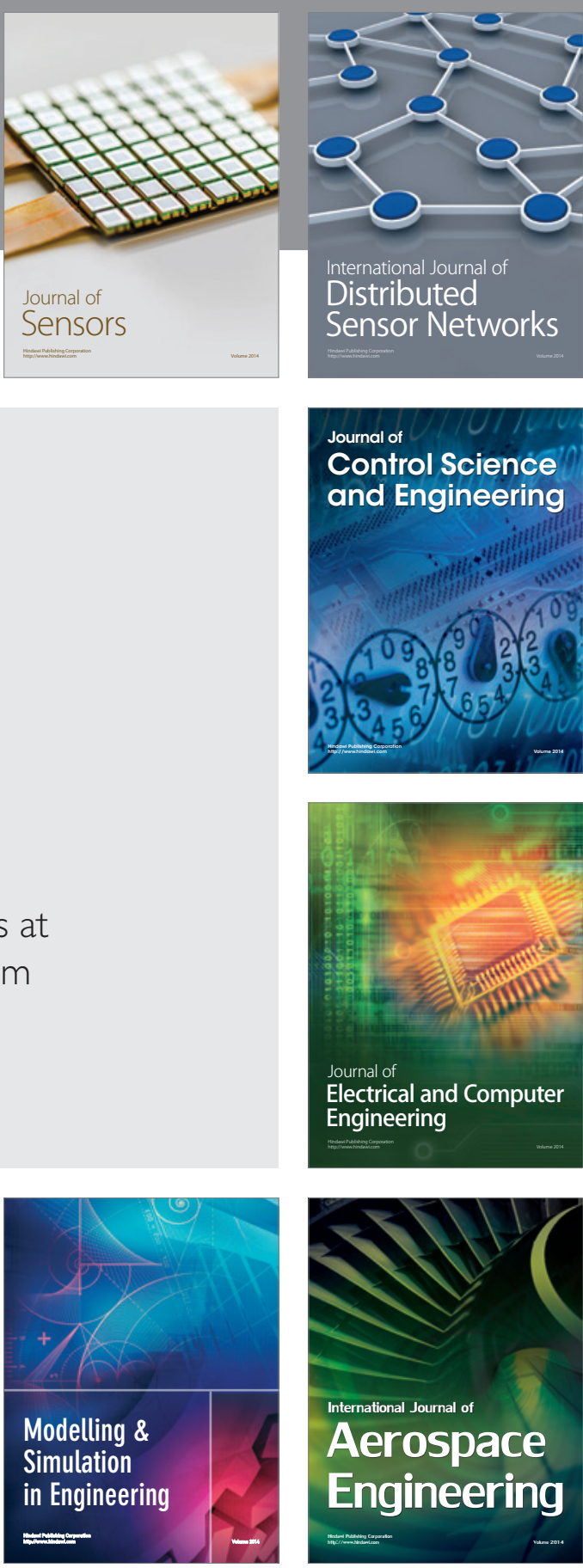

International Journal of

Distributed

Sensor Networks

$-$

Joumal of

Control Science

and Engineering
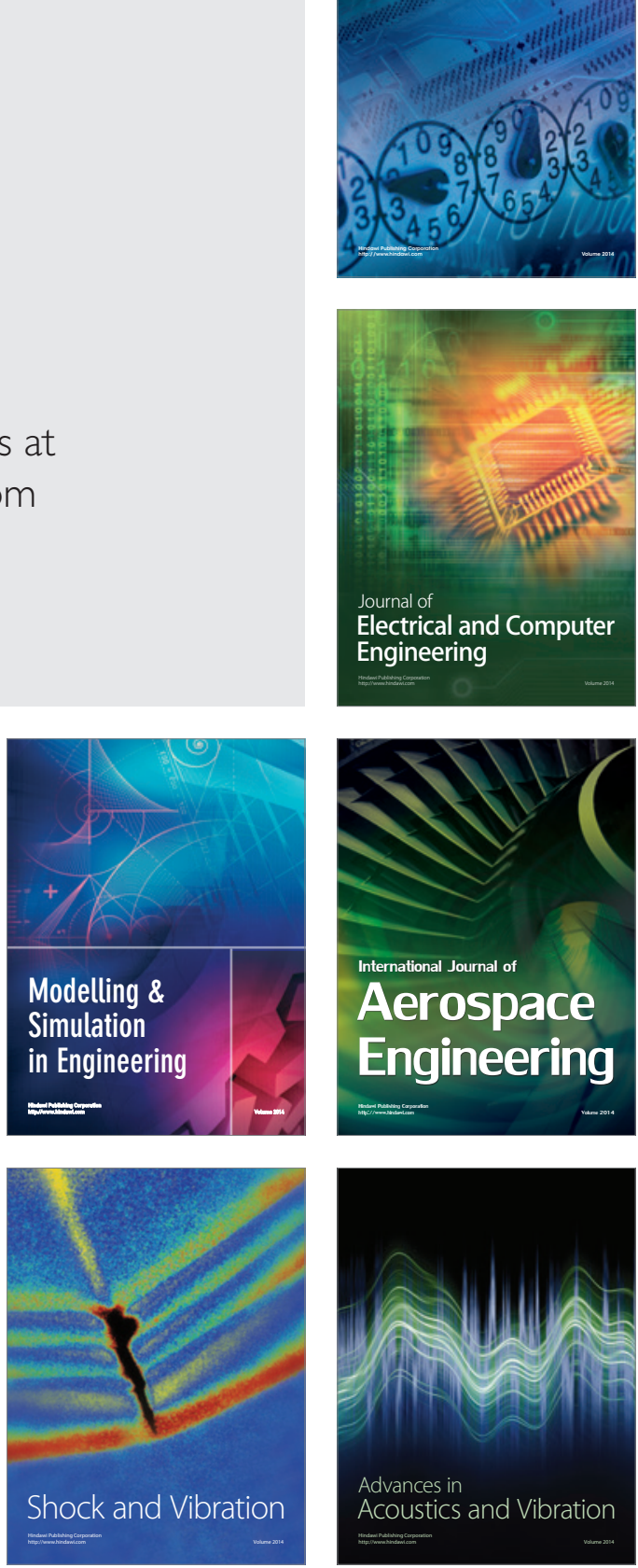